CALT-68-2723

\title{
THE SCIENTIFIC CONTRIBUTIONS OF JOËL SCHERK
}

\author{
John H. Schwarz \\ California Institute of Technology \\ Pasadena, CA 91125, USA
}

\begin{abstract}
Joël Scherk (1946-1980) was an important early contributor to the development of string theory. Together with various collaborators, he made numerous profound and influential contributions to the subject throughout the decade of the 1970s. On the occasion of a conference at the École Normale Supérieure in 2000 that was dedicated to the memory of Joël Scherk I gave a talk entitled "Reminiscences of Collaborations with Joël Scherk" [1]. The present article, an expanded version of that one, also discusses work in which I was not involved.
\end{abstract}

Contribution to a volume entitled The Birth of String Theory 


\section{Introduction}

Joël Scherk and André Neveu were two brilliant French theoretical physicists who emerged in the latter part of the 1960s. They were students together at the elite École Normale Supérieure in Paris and in Orsay, where they studied electromagnetic and final-state interaction corrections to nonleptonic kaon decays [2] under the guidance of Claude Bouchiat and Philippe Meyer. They defended their "thèse de troisième cycle" (the French equivalent of a $\mathrm{PhD}$ ) in 1969, and they were hired together by the CNRS that year [3]. (Tenure at age 23!) In September 1969 the two of them headed off to Princeton University. Joël was supported by a NATO Fellowship 1

In 1969 my duties as an Assistant Professor in Princeton included advising some assigned graduate students. The first advisees, who came together to see me, were André Neveu and Joël Scherk. I had no advance warning about them, and so I presumed they were just another pair of entering students. I certainly didn't know that they had permanent jobs in France. Because their degrees were not called PhDs, Princeton University classified them as graduate students, and so (by the luck of the draw) they were assigned to me. At our first meeting, I asked the usual questions: "Do you need to take a course on electrodynamics?", "Do you need to take a course on quantum mechanics?", etc. They assured me that they already had learned all that, so it wouldn't be necessary. I said okay, signed their cards, and they left.

\section{Loop Amplitudes}

Veneziano discovered his famous formula for a four-particle amplitude in 1968 [4. In 1969 various groups constructed $N$-particle generalizations of the Veneziano amplitude [5]-[9] and showed that they could be consistently factorized on a well-defined spectrum of singleparticle states as required for the tree approximation of a quantum theory [10]-[13]. In those days the theory in question was called the dual resonance model. Today we would refer to it as the bosonic string theory. Knowing the tree-approximation spectrum and couplings, it became possible to construct one-loop amplitudes. The first such attempt was made by Kikkawa, Sakita, and Virasoro [14. They did not have enough information in hand to do it completely right, but they pioneered many of the key ideas and pointed the way for their successors. Around this time (the fall of 1969) I began studying these one-loop amplitudes in collaboration with David Gross, who was also an Assistant Professor at Princeton.

A couple months after our first meeting, Joël and André reappeared in my office and

\footnotetext{
${ }^{1}$ President De Gaulle had pulled France out of the military part of NATO in 1966, but not out of the educational/scientific/cultural part. See [3] for an amusing account of how Joël learned that NATO support was possible.
} 
said that they had found some results they would like to show me. They proceeded to explain their analysis of the divergence in the planar one-loop amplitude. They had realized that by performing a Jacobi transformation of the theta functions in the integrand they could isolate the divergent piece and propose a fairly natural counterterm [15]. I was very impressed by this achievement. It certainly convinced me that they did not need to take any more quantum mechanics courses! The modern interpretation of their result is that, viewed in a dual channel, there is a closed string going into the vacuum. The divergence can be attributed to the tachyon in that channel, and its contribution is the piece that they subtracted. This interpretation explains why such divergences do not occur in theories without closed-string tachyons.

Since André and Joël were working on problems that were very closely related to those that David and I were studying, we decided to join forces. Even though we were not yet thinking in terms of string world sheets, we were able to relate the classification of dual resonance model amplitudes to the topological classification of Riemann surfaces with boundaries [16]. Another discovery by the four of us was that the nonplanar loop amplitude contains unexpected singularities [17]. These appeared in addition to the expected two-particle threshold singularities 2 We assumed, of course, that the dimension of spacetime is four, since nobody had yet suggested otherwise. The Virasoro constraints, which should be taken into account for the internal states circulating in the loop, had not yet been discovered. As a result, the singularities that we found were unitarity-violating branch points. We wanted to identify the leading Regge trajectory associated to these singularities with the Pomeron, since it carried vacuum quantum numbers, but clearly something wasn't quite right.

About a year later Lovelace observed that if one chooses the spacetime dimension to be 26 (one time and 25 space) and supposes that the Virasoro conditions imply that only transverse excitations contribute, then instead of branch points the singularities would be poles [19], which could be interpreted as new states in the spectrum without violating unitarity. As we now know, these are the closed-string states in the nonplanar open-string loop. Nowadays this is interpreted as open-string closed-string duality of the cylinder diagram. This calculation showed that unitarity requires choosing the dimension to be 26 and the Regge intercept value to be one, since these are requirements for the Virasoro conditions to be satisfied.

Joël left Princeton in the spring of 1970 to spend about six months in Berkeley. While he was there he collaborated with Michio Kaku, who was a student of Stanley Mandelstam at the time, studying divergences in multiloop planar amplitudes [20, 21]. This was a very ambitious project, given the state of the art in those days.

\footnotetext{
${ }^{2}$ This was discovered independently by Frye and Susskind at about the same time [18.
} 


\section{Orsay, CERN, and NYU}

Scherk's NATO Fellowship was only good for one year, so following his visits to Princeton and Berkeley, he returned to Orsay for a year followed by two years at CERN. While he was at Orsay, Joël pioneered the idea of considering string theories (or dual models) in a zero-slope limit, which is equivalent to a low-energy limit [22]. In particular, Joël and André studied the massless open-string spin-one states and showed that in a suitable low-energy limit they interacted precisely in agreement with Yang-Mills theory [23], and they studied the associated gauge invariance that this implied for the full string theory [24]. These studies made it clear that open strings and their interactions could be viewed as short-distance modifications of Yang-Mills theory. This important observation certainly influenced the future evolution of the subject.

During the two-year period that Joël spent at CERN, beginning in August 1971, he shared an office with Lars Brink. At first, he collaborated with Eugène Cremmer on the study of currents and off-shell amplitudes in string theory as well as factorization in the closed string (or "Pomeron") sector [25, 26, 27]. After that, he collaborated with Lars Brink, David Olive, and Claudio Rebbi on the study of scattering amplitudes for Ramond fermions [29, 30].

By now, Joël was very well-known and in much demand. He decided to accept invitations from NYU and Caltech for the academic year 1973-74, spending the Fall term at NYU and the Winter and Spring terms at Caltech. During his visit to NYU, he wrote a very elegant and highly-cited review of string theory for the Reviews of Modern Physics [31]. This article was based on a course that he taught at NYU. He also completed a paper with Eugène concerning gauge symmetry breaking [32] in the string theory context.

\section{String Theory for Unification}

In 1972 I left Princeton and moved to Caltech. At Caltech, Murray Gell-Mann provided funds for me to invite collaborators of my choosing. One of them was Joël Scherk, who spent the first half of 1974 visiting Caltech. The timing couldn't have been better.

The hadronic interpretation of string theories was plagued not only by the occurrence of massless vector particles in the open-string spectrum, but by a massless tensor particle in the closed-string spectrum, as well. Several years of effort were expended on trying to modify each of the two string theories (bosonic and RNS) so as to lower the leading open-string Regge intercept from 1 to $1 / 2$ and the leading closed-string Regge intercept from 2 to 1 , since these were the values required for the leading meson and Pomeron Regge trajectories. Some partial successes were achieved, but no fully consistent scheme was found. Efforts to 
modify the critical spacetime dimension from 26 or 10 to four also led to difficulties.

By 1974, almost everyone who had been working on string theory had dropped it and moved to greener pastures. The standard model had been developed, and it was working splendidly. Against this backdrop, Joël and I stubbornly decided to return to the nagging unresolved problems of string theory. We felt that string theory has such a compelling mathematical structure that it ought to be good for something. Before long our focus shifted to the question of whether the massless spin two particle in the spectrum interacted at low energies in accordance with the dictates of general relativity, so that it might be identified as a graviton. As was mentioned previously, Joël and André Neveu had studied the massless open-string spin-one states a few years earlier and showed that in a suitable low-energy limit they interacted precisely in agreement with Yang-Mills theory [22, 23]. Now we wondered about the analogous question for the massless spin-two closed-string state. Roughly, what we proved was that critical string theories have the gauge invariances required to decouple unphysical polarization states [34]. Then it followed on general grounds, which had been elaborated previously by Weinberg [35], that the interactions at low energy must be those of general relativity.

Once we had digested the fact that string theory inevitably contains gravity we were very excited. We knew that string theory does not have ultraviolet divergences, because the short-distance structure is smoothed out, but any field-theoretic approach to gravitation inevitably gives nonrenormalizable ultraviolet divergences. Evidently, the way to make a consistent quantum theory of gravity is to posit that the fundamental entities are strings rather than point particles [34] 3

Adopting this viewpoint meant that the fundamental length scale of string theory, called the string scale, should be identified with the Planck scale in order to give the correct value for Newton's constant, at least if one assumes that the size of the extra dimensions is also given by the string scale. The Planck scale thus replaces the QCD scale, which was the natural choice for the string scale when string theory was being developed as a framework for describing strong interactions (hadron physics). This is a change of some 19 or 20 orders of magnitude. Even though the mathematics was largely unchanged, this was a large conceptual leap. Convinced of the importance of this viewpoint, we submitted a short essay summarizing the argument to the Gravity Research Foundation's 1975 Essay competition [37. The judges were not very impressed, so we only received an Honorable Mention.

Our 1974 paper proposed changing the goal of string theory to the problem of constructing a consistent quantum theory of gravity. Since we already knew from the earlier work of André and Joël that string theory also contains Yang-Mills gauge interactions, it was natural for

\footnotetext{
${ }^{3}$ For a more detailed discussion see [33] in this volume.
} 
us to propose further that string theory should describe all the other forces at the same time. This means interpreting string theory as a unified quantum theory of all fundamental particles and forces - an explicit realization of Einstein's dream. Moreover, we realized that the existence of extra dimensions could now be a blessing rather than a curse. After all, in a gravity theory the geometry of spacetime is determined dynamically, and one could imagine that this would require, or at least allow, the extra dimensions to form a very small compact space. We attempted to construct a specific compactification scenario in a subsequent paper [38]. From today's vantage point, that construction looks rather primitive.

Tamiaki Yoneya independently realized that the massless spin two state of string theory interacts at low energy in accordance with the dictates of general relativity [39]. Indeed, his paper appeared first, though we were not aware of it at the time. However, as Yoneya graciously acknowledges, Joël and I were the only ones to take the next step and to propose that string theory should be the basis for constructing a unified quantum theory of all forces. The recognition of that possibility represented a turning point in my research career. I found the case compelling, and I became committed to exploring its implications. I believe that Joël felt the same way. I still do not understand why it took another decade until a large segment of the theoretical physics world became convinced that string theory was the right approach to unification. (There were some people who caught on earlier, of course.) One of my greatest regrets is that Joël was not able to witness the impact that this idea eventually would have.

During Joël's Caltech visit, we also explored some aspects of gravity that could be affected by the string interpretation. One paper interpreted the three-form flux $H=d B$ in terms of spacetime torsion [40]. Since we knew that the light-cone gauge is convenient for exploring certain aspects of string theory, we also attempted to formulate general relativity in the light-cone gauge [41].

After leaving Caltech, Joël participated in a summer 1974 workshop on string theory at the Aspen Center for Physics, which I organized. This had been planned a year earlier, when there were still quite a few people working in string theory. My memory is fading, but I do not recall the participants showing much interest in our proposal to use string theory for unification.

After Aspen, Joël returned to Paris, since his group at Orsay had moved from Orsay to the Laboratoire de Physique Théorique of the École Normale Supérieure in Paris. Aside from a few months in Cambridge in 1977 and brief visits elsewhere, this is where Joël spent the remainder of his career. At the LPTENS, Joël resumed his collaboration with Eugène Cremmer. Soon, they turned out a pair of well-known papers [42, 43] that grappled with issues raised by the unification interpretation of string theory. The first paper [42] introduced 
the notion of winding numbers for the first time. As is now well-known, closed strings can wrap on cycles in the compact dimensions. This possibility was important in the later construction of the heterotic string as well as for the discovery of T-duality almost a decade later 4 The second paper [43] emphasized the idea that the compact internal spaces cannot be chosen arbitrarily; instead, they are fixed by the mechanism that they called "spontaneous compactification". This means that they must be stable or metastable solutions of the equations of motion 5

\section{$5 \quad$ Spacetime Supersymmetry}

The super-Virasoro gauge symmetry of the Ramond-Neveu-Schwarz model [45, 46] describes the superconformal symmetry of the two-dimensional world-sheet theory. The supersymmetry of the two-dimensional world-sheet action was described later in 1971 by Gervais and Sakita [47]. This was the first example of a supersymmetric quantum field theory. For about five years, the supersymmetry considered by string theorists only pertained to the two-dimensional world-sheet theory. It did not occur to us that there could also be supersymmetry in ten-dimensional spacetime.

Bruno Zumino, who was also at CERN when Joël was there, became very interested in the RNS string's gauge conditions associated to the two-dimensional superconformal algebra and discussed it at length with Joël and Lars. His work on this subject is described in [48]. Following that, he and Julius Wess began to consider the possibility of constructing four-dimensional field theories with analogous features. This resulted in their famous work [49] on globally supersymmetric field theories in four dimensions. As a consequence of their paper 6 supersymmetry quickly became an active research topic. A few years later came the discovery of supergravity theories [51, 52].

Lars Brink, Joël, and I constructed supersymmetric Yang-Mills theories in various dimensions [54]. When this work was carried out, Lars and I were at Caltech and Joël was in Paris. (We communicated by mail, since email was not yet an option.) We discovered that the requisite gamma-matrix identity required by these theories, $\gamma_{(a b}^{m} \gamma_{c) d}^{m}=0$, is satisfied in dimensions 3, 4, 6, and 10. Dimensional reduction was also discussed, giving (among other things) the first construction of $\mathcal{N}=4$ super Yang-Mills theory in four dimensions. At the same time as the work described above, Joël was collaborating with Fernando Gliozzi and

\footnotetext{
${ }^{4}$ Eugène informs me that they failed to discover T-duality because of their field theory prejudices. This shows that even dedicated string theorists had such prejudices. See 44 for further discussion of this.

${ }^{5}$ Metastability implies massless moduli, which are ruled out experimentally by tests of the tensor nature of the gravitational force.

${ }^{6}$ The work of Golfand and Likhtman [50, which was the first to introduce the four-dimensional superPoincaré group, was not known in the West at that time.
} 
David Olive on some closely related ideas [55, [56]. This threesome is now referred to as GSO. (Lars and I were not aware of this collaboration until we saw their papers.) The GSO papers also explored super Yang-Mills theories. Moreover, they took the next major step, which concerned the RNS string theory.

GSO proposed a projection of the RNS spectrum - the GSO Projection - that removes roughly half of the states (including the tachyon). Specifically, in the bosonic (NS) sector they projected away the odd G-parity states, a possibility that was discussed earlier, and in the fermionic $(\mathrm{R})$ sector they projected away half the states, keeping only certain definite chiralities. Then they counted the remaining physical degrees of freedom of the free string at each mass level. After the GSO projection the masses of open-string states, for both bosons and fermions, are given by $\alpha^{\prime} M^{2}=n$, where $n=0,1,2, \ldots$ Denoting the open-string degeneracies of states in the GSO-projected theory by $d_{\mathrm{NS}}(n)$ and $d_{\mathrm{R}}(n)$, they showed that these are encoded in the generating functions

$$
\begin{gathered}
f_{\mathrm{NS}}(w)=\sum_{n=0}^{\infty} d_{\mathrm{NS}}(n) w^{n} \\
=\frac{1}{2 \sqrt{w}}\left[\prod_{m=1}^{\infty}\left(\frac{1+w^{m-1 / 2}}{1-w^{m}}\right)^{8}-\prod_{m=1}^{\infty}\left(\frac{1-w^{m-1 / 2}}{1-w^{m}}\right)^{8}\right] .
\end{gathered}
$$

and

$$
f_{\mathrm{R}}(w)=\sum_{n=0}^{\infty} d_{\mathrm{R}}(n) w^{n}=8 \prod_{m=1}^{\infty}\left(\frac{1+w^{m}}{1-w^{m}}\right)^{8} .
$$

In 1829, Jacobi proved the remarkable identity [58]

$$
f_{\mathrm{NS}}(w)=f_{\mathrm{R}}(w)
$$

though he used a different notation 7 Thus, there are an equal number of bosons and fermions at every mass level, as required. This was compelling evidence (though not a proof) for tendimensional spacetime supersymmetry of the GSO-projected theory. Prior to this work, one knew that the RNS theory has world-sheet supersymmetry, but the realization that the theory should have spacetime supersymmetry was a major advance. I was very delighted by this result. One could now envisage a tachyon-free string theory that would make sense as a starting point for a unified theory.

At about the same time as the GSO breakthrough, Joël collaborated with Sergio Ferrara, Dan Freedman, Peter Van Nieuwenhuizen, and Bruno Zumino on various different topics in supergravity [57]-61]. In the following year (1977) he continued studying supergravity, now collaborating mostly with Eugène [62]-67]. Two of these papers [64, 65] were written while

\footnotetext{
${ }^{7}$ Jacobi's paper acknowledges an assistant named Scherk!
} 
Joël spent several months in the Spring of 1977 at the DAMTP in Cambridge. Eugène and Joël communicated by snail mail and FAX during that period.

Eugène, Sergio, and Joël reformulated $\mathcal{N}=4$ supergravity in a manifestly $S U(4)$ invariant form that was motivated by $N=1$ supergravity in ten dimensions, which was itself motivated by superstring theory. It exhibited an on-shell $S U(1,1)$ duality invariance [66]. This was the first discovery of the duality symmetries of extended supergravity theories. S-duality in string theory is such a duality, as was understood some 17 years later. The discovery of such dualities was very influential in the construction of $\mathcal{N}=8$ supergravity by Eugène and Bernard Julia [69, 70]. The duality group in that case is $E_{7,7}$. Eugène informs me that health problems prevented Joël from participating in that collaboration.

One of the most beautiful results in supergravity, and Joël's most-cited paper, is the March 1978 construction of the action of 11-dimensional supergravity by Eugène, Bernard, and Joël [68]. It was immediately clear that 11-dimensional supergravity is very beautiful, and it aroused a lot of interest. However, it was puzzling for a long time how it fits into the greater scheme of things and whether it has any connection to string theory. Clearly, supergravity in 11 dimensions is not a consistent quantum theory by itself, since it is very singular in the ultraviolet. Moreover, since superstring theory only has ten dimensions, it did not seem possible that it could serve as a regulator. It took more than fifteen years to find the answer to this conundrum [71, 72]: At strong coupling Type IIA superstring theory develops a circular 11th dimension whose radius grows with the string coupling constant. In the limit of infinite coupling one obtains $M$ theory, which is presumably a well-defined quantum theory that has 11 noncompact dimensions. Eleven-dimensional supergravity is the leading low-energy approximation to $\mathrm{M}$ theory. In other words, $\mathrm{M}$ theory is the UV completion of 11-dimensional supergravity.

Later in 1978 Eugène, Bernard, and Joël teamed up with Sergio Ferrara, Luciano Girardello, and Peter Van Nieuwenhuizen on a pair of papers studying the super Higgs effect in four-dimensional supergravity theories coupled to matter [73, 74]. This work has been used a great deal in subsequent studies.

\section{$6 \quad$ Supersymmetry Breaking}

I spent the academic year 1978-79 visiting the LPTENS, on leave of absence from Caltech. I was eager to work with Joël on supergravity, supersymmetrical strings, and related matters. He was struggling with rather serious health problems during that year, so he wasn't able to participate as fully as when we were in Caltech five years earlier, but he was able to work about half time. On that basis we were able to collaborate successfully. 
After various wide-ranging discussions we decided to focus on the problem of supersymmetry breaking. We wondered how, starting from a supersymmetric string theory in ten dimensions, one could end up with a nonsupersymmetric world in four dimensions. The specific supersymmetry breaking mechanism that we discovered can be explained classically and does not really require strings, so we explored it in a field theoretic setting [75, 76]. The idea is that in a theory with extra dimensions and global symmetries that do not commute with supersymmetry ( $R$ symmetries and $(-1)^{F}$ are examples), one could arrange for a twisted compactification, and that this would break supersymmetry. For example, if one extra dimension forms a circle, the fields when continued around the circle could come could back transformed by an R-symmetry group element. If the gravitino, in particular, is transformed then it acquires mass in a consistent manner.

An interesting example of our supersymmetry breaking mechanism was worked out in a paper we wrote together with Eugène [77]. We started with maximal supergravity in five dimensions. This theory contains eight gravitinos that transform in the fundamental representation of a $\mathrm{USp}(8)$ R-symmetry group. We took one dimension to form a circle and examined the resulting four-dimensional theory keeping the lowest Kaluza-Klein modes. The supersymmetry-breaking R-symmetry element is a $\operatorname{USp}(8)$ element that is characterized by four real mass parameters, since this group has rank four. These four masses give the masses of the four complex gravitinos of the resulting four-dimensional theory. In this way we were able to find a consistent four-parameter deformation of $\mathcal{N}=8$ supergravity.

Even though the work that Joël and I did on supersymmetry breaking was motivated by string theory, we only discussed field theory applications in our articles. The reason I never wrote about string theory applications was that in the string theory setting it did not seem possible to decouple the supersymmetry breaking mass parameters from the compactification scales. This was viewed as a serious problem, because the two scales are supposed to be hierarchically different. In recent times, people have been considering string theory braneworld scenarios in which much larger compactification scales are considered. In such a context our supersymmetry breaking mechanism might have a role to play. Indeed, quite a few authors have explored various such possibilities.

\section{Concluding Comments}

When I left Paris in the summer of 1979 I visited CERN for a month. There I began a collaboration with Michael Green. During that month we began to formulate a plan for exploring how the spacetime supersymmetry identified by GSO is realized in the interacting string theory. In September 1979, when I spoke at a conference on supergravity that was 
held in Stony Brook, we did not yet have definitive results. Therefore, I reported on the work that Joël and I had done on supersymmetry breaking [78]. Joël gave a talk entitled From Supergravity to Antigravity at the Stony Brook conference [79, 80]. He was intrigued by the fact that graviton exchanges in string theory are accompanied by antisymmetric tensor and scalar exchanges that can cancel the gravitational attraction. Nowadays we understand that the effect that he was discussing is quite important. For example, parallel BPS D-branes form stable supersymmetric systems precisely because the various forces cancel. The Stony Brook conference was the last time that I saw Joël.

In March 1980 Joël attended a meeting in Erice, Sicily. Lars Brink, who was also there, recalls being very worried about Joël's health. Six weeks after that meeting he passed away, which came as a great shock to his many friends and colleagues. The ideas that Joël pioneered during the decade of the 1970s have been very influential in the subsequent decades. It is a

pity that he could not participate in these developments and enjoy the recognition that he would have received.

\section{Acknowledgments}

I am grateful to Lars Brink, Eugène Cremmer, André Neveu, and David Olive for reading this manuscript and making numerous helpful suggestions. This work was supported in part by the U.S. Dept. of Energy under Grant No. DE-FG03-92-ER40701. 


\section{References}

[1] J. H. Schwarz, "Reminiscences of Collaborations with Joël Scherk," arXiv:hep-th/0007117.

[2] A. Neveu and J. Scherk, "Final-State Interaction and Current Algebra in $K \rightarrow 3 \pi$ and $\eta \rightarrow 3 \pi$ Decays," Annals Phys. 57 (1970) 39.

[3] A. Neveu, "Personal Recollections about the First Three Years of String Theory," this volume.

[4] G. Veneziano, "Construction of a Crossing-Symmetric Regge-Behaved Amplitude for Linearly Rising Regge Trajectories," Nuovo Cim. 57A (1968) 190.

[5] K. Bardakci and H. Ruegg, "Reggeized Resonance Model for Arbitrary Production Processes," Phys. Rev. 181 (1969) 1884.

[6] C. J. Goebel and B. Sakita, "Extension of the Veneziano Formula to $N$-Particle Amplitudes," Phys. Rev. Lett. 22 (1969) 257.

[7] H. M. Chan and T. S. Tsun, "Explicit Construction of the $N$-Point Function in the Generalized Veneziano Model," Phys. Lett. 28B (1969) 485.

[8] Z. Koba and H. B. Nielsen, "Reaction Amplitudes for $N$ Mesons, a Generalization of the Veneziano-Bardakci-Ruegg-Virasoro Model," Nucl. Phys. B10 (1969) 633.

[9] Z. Koba and H. B. Nielsen, "Manifestly Crossing-Invariant Parametrization of the $\mathrm{N}$ Meson Amplitude," Nucl. Phys. B12 (1969) 517.

[10] S. Fubini and G. Veneziano, "Level Structure of Dual Resonance Models," Nuovo Cim. 64A (1969) 811.

[11] S. Fubini, D. Gordon, and G. Veneziano, "A General Treatment of Factorization in Dual Resonance Models," Phys. Lett. 29B (1969) 679.

[12] K. Bardakci and S. Mandelstam, "Analytic Solution of the Linear-Trajectory Bootstrap," Phys. Rev. 184 (1969) 1640.

[13] S. Fubini and G. Veneziano, "Duality in Operator Formalism," Nuovo Cim. 67A (1970) 29.

[14] K. Kikkawa, B. Sakita and M. A. Virasoro, "Feynman-like diagrams compatible with duality. I: Planar diagrams," Phys. Rev. 184 (1969) 1701. 
[15] A. Neveu and J. Scherk, "Parameter-Free Regularization of One-Loop Unitary Dual Diagram," Phys. Rev. D1 (1970) 2355.

[16] D. J. Gross, A. Neveu, J. Scherk, and J. H. Schwarz, "The Primitive Graphs of Dual Resonance Models," Phys. Lett. 31B (1970) 592.

[17] D. J. Gross, A. Neveu, J. Scherk, and J. H. Schwarz, "Renormalization and Unitarity in the Dual Resonance Model," Phys. Rev. D2 (1970) 697.

[18] G. Frye and L. Susskind, "Non-Planar Dual Symmetric Loop Graphs and the Pomeron," Phys. Lett. 31B (1970) 589.

[19] C. Lovelace, "Pomeron Form Factors and Dual Regge Cuts," Phys. Lett. 34B (1971) 500.

[20] M. Kaku and J. Scherk, "Divergence of the Two-Loop Planar Graph in the DualResonance Model," Phys. Rev. D 3 (1971) 430.

[21] M. Kaku and J. Scherk, "Divergence of the N-Loop Planar Graph in the Dual-Resonance Model," Phys. Rev. D 3 (1971) 2000.

[22] J. Scherk, "Zero Slope Limit of the Dual Resonance Model," Nucl. Phys. B31 (1971) 222.

[23] A. Neveu and J. Scherk, "Connection Between Yang-Mills Fields and Dual Models," Nucl. Phys. B36 (1972) 155.

[24] A. Neveu and J. Scherk, "Gauge Invariance and Uniqueness of the Renormalisation of Dual Models with Unit Intercept," Nucl. Phys. B 36 (1972) 317.

[25] E. Cremmer and J. Scherk, "Currents and Green's Functions for Dual Models. (II) Off-Shell Dual Amplitudes," Nucl. Phys. B 48 (1972) 29.

[26] E. Cremmer and J. Scherk, "Factorization of the Pomeron Sector and Currents in the Dual Resonance Model," Nucl. Phys. B50 (1972) 222.

[27] E. Cremmer and J. Scherk, "Regge Limit and Scaling in a Dual Model of Currents," Nucl. Phys. B 58 (1973) 557.

[28] L. Brink, D. I. Olive and J. Scherk, "The Gauge Properties of the Dual Model PomeronReggeon Vertex - Their Derivation and Their Consequences," Nucl. Phys. B 61 (1973) 173. 
[29] D. I. Olive and J. Scherk, "Towards Satisfactory Scattering Amplitudes for Dual Fermions," Nucl. Phys. B 64 (1973) 334.

[30] L. Brink, D. I. Olive, C. Rebbi and J. Scherk, "The Missing Gauge Conditions for the Dual Fermion Emission Vertex and Their Consequences," Phys. Lett. B 45 (1973) 379.

[31] J. Scherk, "An Introduction to the Theory of Dual Models and Strings," Rev. Mod. Phys. 47 (1975) 123.

[32] E. Cremmer and J. Scherk, "Spontaneous Dynamical Breaking of Gauge Symmetry in Dual Models," Nucl. Phys. B 72 (1974) 117.

[33] J. H. Schwarz, The Early Years of String Theory: A Personal Perspective," in this volume.

[34] J. Scherk and J. H. Schwarz, "Dual Models for Non-Hadrons," Nucl. Phys. B81 (1974) 118.

[35] S. Weinberg, "Photons and Gravitons in Perturbation Theory: Derivation of Maxwell's and Einstein's Equations," Phys. Rev. 138 (1965) B988.

[36] J. Scherk and J. H. Schwarz, "Dual Models and the Geometry of Space-Time," Phys. Lett. B52 (1974) 347.

[37] J. Scherk and J. H. Schwarz, "Dual Model Approach to a Renormalizable Theory of Gravitation," Submitted to the 1975 Gravitation Essay Contest of the Gravity Research Foundation. Reprinted in Superstrings, Vol. 1, ed. J. Schwarz, World Scientific (1985).

[38] J. Scherk and J. H. Schwarz, "Dual Field Theory of Quarks and Gluons," Phys. Lett. 57B (1975) 463.

[39] T. Yoneya, "Connection of Dual Models to Electrodynamics and Gravidynamics," Prog. Theor. Phys. 51 (1974) 1907.

[40] J. Scherk and J. H. Schwarz, "Dual Models and the Geometry of Space-Time," Phys. Lett. B 52 (1974) 347.

[41] J. Scherk and J. H. Schwarz, "Gravitation in the Light-Cone Gauge," Gen. Rel. Grav. 6 (1975) 537.

[42] E. Cremmer and J. Scherk, "Dual Models in Four Dimensions with Internal Symmetries," Nucl. Phys. B 103 (1976) 399. 
[43] E. Cremmer and J. Scherk, "Spontaneous Compactification of Space in an Einstein Yang-Mills Higgs Model," Nucl. Phys. B 108 (1976) 409.

[44] E. Cremmer, "Person Recollections about the Birth of String Theory," this volume.

[45] P. Ramond, "Dual Theory for Free Fermions," Phys. Rev, D 3 (1971) 2415.

[46] A. Neveu and J. H. Schwarz, "Factorizable Dual Model of Pions," Nucl. Phys. B 31 (1971) 86.

[47] J. L. Gervais and B. Sakita, "Field Theory Interpretation of Supergauges in Dual Models," Nucl. Phys. B 34 (1971) 632.

[48] B. Zumino, "Relativistic Strings and Supergauges," p. 367 in Renormalization and Invariance in Quantum Field Theory, ed. E. Caianiello (Plenum Press, 1974).

[49] J. Wess and B. Zumino, "Supergauge Transformations in Four-Dimensions," Nucl. Phys. B 70 (1974) 39.

[50] Yu. A. Golfand and E. P. Likhtman, "Extension of the Algebra of Poincaré Group Generators and Violation of P Invariance," JETP Lett. 13 (1971) 323 [Pisma Zh. Eksp. Teor. Fiz. 13 (1971) 452].

[51] D. Z. Freedman, P. van Nieuwenhuizen and S. Ferrara, "Progress Toward a Theory of Supergravity," Phys. Rev. D 13 (1976) 3214.

[52] S. Deser and B. Zumino, "Consistent Supergravity," Phys. Lett. B 62 (1976) 335.

[53] S. Ferrara, J. Scherk and P. van Nieuwenhuizen, Phys. Rev. Lett. 37 (1976) 1035.

[54] L. Brink, J. H. Schwarz, and J. Scherk , "Supersymmetric Yang-Mills Theories," Nucl. Phys. B121, 77 (1977).

[55] F. Gliozzi, J. Scherk, and D. Olive, 'Supergravity and the Spinor Dual Model," Phys. Lett. 65B, 282 (1976).

[56] F. Gliozzi, J. Scherk, and D. Olive, "Supersymmetry, Supergravity Theories and the Dual Spinor Model," Nucl. Phys. B122, 253 (1977).

[57] S. Ferrara, D. Z. Freedman, P. van Nieuwenhuizen, P. Breitenlohner, F. Gliozzi and J. Scherk, "Scalar Multiplet Coupled to Supergravity," Phys. Rev. D 15 (1977) 1013.

[58] C. G. J. Jacobi, Fundamenta, Konigsberg, 1829. 
[59] S. Ferrara, F. Gliozzi, J. Scherk and P. Van Nieuwenhuizen, "Matter Couplings in Supergravity Theory," Nucl. Phys. B 117 (1976) 333.

[60] S. Ferrara, J. Scherk and B. Zumino, "Supergravity and Local Extended Supersymmetry," Phys. Lett. B 66 (1977) 35.

[61] S. Ferrara, J. Scherk and B. Zumino, "Algebraic Properties of Extended Supergravity Theories," Nucl. Phys. B 121 (1977) 393.

[62] E. Cremmer, J. Scherk and S. Ferrara, "U(N) Invariance in Extended Supergravity," Phys. Lett. B 68 (1977) 234.

[63] Z. Horvath, L. Palla, E. Cremmer and J. Scherk, "Grand Unified Schemes and Spontaneous Compactification," Nucl. Phys. B 127 (1977) 57.

[64] E. Cremmer and J. Scherk, "Algebraic Simplifications in Supergravity Theories," Nucl. Phys. B 127 (1977) 259.

[65] E. Cremmer and J. Scherk, "Modified Interaction of the Scalar Multiplet Coupled to Supergravity," Phys. Lett. B 69 (1977) 97.

[66] E. Cremmer, J. Scherk and S. Ferrara, "SU(4) Invariant Supergravity Theory," Phys. Lett. B 74 (1978) 61.

[67] E. Cremmer and J. Scherk, "The Supersymmetric Nonlinear Sigma Model in FourDimensions and Its Coupling to Supergravity," Phys. Lett. B 74 (1978) 341.

[68] E. Cremmer, B. Julia, and J. Scherk, "Supergravity Theory in 11 Dimensions," Phys. Lett. 76B (1978) 409.

[69] E. Cremmer and B. Julia, "The N=8 Supergravity Theory. 1. The Lagrangian," Phys. Lett. B 80 (1978) 48.

[70] E. Cremmer and B. Julia, "The SO(8) Supergravity," Nucl. Phys. B 159 (1979) 141.

[71] P. K. Townsend, "The Eleven-Dimensional Supermembrane Revisited," Phys. Lett. B 350 (1995) 184 arXiv:hep-th/9501068.

[72] E. Witten, "String Theory Dynamics in Various Dimensions," Nucl. Phys. B 443 (1995) 85 arXiv:hep-th/9503124. 
[73] E. Cremmer, B. Julia, J. Scherk, P. van Nieuwenhuizen, S. Ferrara and L. Girardello, "Superhiggs Effect in Supergravity with General Scalar Interactions," Phys. Lett. B 79 (1978) 231.

[74] E. Cremmer, B. Julia, J. Scherk, S. Ferrara, L. Girardello and P. van Nieuwenhuizen, "Spontaneous Symmetry Breaking and Higgs Effect in Supergravity Without Cosmological Constant," Nucl. Phys. B 147 (1979) 105.

[75] J. Scherk and J. H. Schwarz, "Spontaneous Breaking of Supersymmetry Through Dimensional Reduction," Phys. Lett. B82, 60 (1979).

[76] J. Scherk and J. H. Schwarz, "How to Get Masses From Extra Dimensions," Nucl. Phys. B153, 61 (1979).

[77] E. Cremmer, J. Scherk and J. H. Schwarz, "Spontaneously Broken N = 8 Supergravity," Phys. Lett. B84, 83 (1979).

[78] J. H. Schwarz, "How to Break Supersymmetry," p. 19 in Supergravity, eds. P. van Nieuwenhuizen and D. Z. Freedman (North-Holland 1979).

[79] J. Scherk, "Antigravity: A Crazy Idea?," Phys. Lett. B 88 (1979) 265.

[80] J. Scherk, "From Supergravity to Antigravity," p. 43 in Supergravity, eds. P. van Nieuwenhuizen and D. Z. Freedman (North-Holland 1979). 